\title{
Hipomelanosis macular progresiva: respuesta al tratamiento con minociclina
}

\section{Progressive macular hypomelanosis: response to treatment with minocycline}

\author{
Fátima Giusti ${ }^{1}$, Carlos Consigli ${ }^{2}$, Javier Consigli ${ }^{3}$, Lorena Cippitelli ${ }^{4}$ y Marco Mazzotta ${ }^{5}$
}

\section{RESUMEN}

La hipomelanosis macular progresiva (HMP) es una dermatosis caracterizada por máculas hipopigmentadas, que se observa con mayor frecuencia en las mujeres y en los fototipos III y IV. Se ha asociado a Cutibacterium acnes (C. acnes) de tipo III como factor etiológico. Se presenta el caso de una paciente de 30 años, con máculas hipopigmentadas redondeadas en el tronco y la raíz de los miembros inferiores, de 10 años de evolución. El estudio histológico informó disminución del número de melanocitos y de pigmento melánico en la capa basal e infiltrado inflamatorio mononuclear perivascular superficial. Se indicó minociclina $100 \mathrm{mg} /$ día por vía oral durante 8 meses, tras lo cual se observó la resolución total de las lesiones.

Palabras clave: hipomelanosis macular progresiva, C. acnes, tetraciclinas, minociclina, luz de Wood.

Dermatol. Argent. 2021, 27 (1): 31-33

\section{ABSTRACT}

Progressive macular hypomelanosis (PMH) is a dermatosis characterized by hypopigmented macules, most frequently found in females and in phototypes III and IV. Cutibacterium acnes (C. acnes) type III has been associated as an etiological factor.

We present the case of a thirty-year-old female patient with a 10year history of nummular hypopigmented macules located on the top of the lower limbs and on the trunk. The histological study reported a decrease in the number of melanocytes and melanotic pigment in the basal layer and the presence of superficial perivascular mononuclear inflammatory infiltrate. After an 8-month regimen of oral minocycline $100 \mathrm{mg} /$ day, there was a complete resolution of the lesions.

Key words: progressive macular hypomelanosis, C. acnes, tetracyclines, minocycline, Wood's light.

Dermatol. Argent. 2021, 27 (1): 31-33

\footnotetext{
1 Médica Concurrente, Servicio de Dermatología

2 Profesor Titular, Servicio de Dermatología

3 Profesor Asociado, Servicio de Dermatología

${ }^{4}$ Profesora Asociada, Servicio de Dermatología

${ }^{5}$ Médico de Planta, Servicio de Anatomía Patológica Hospital Nacional de Clínicas, Facultad de Ciencias Médicas, Universidad Nacional de Córdoba, Córdoba, Argentina
}

Contacto del autor: Fátima Giusti

E-mail: fatigiusti@gmail.com

Fecha de trabajo recibido: $26 / 2 / 2021$

Fecha de trabajo aceptado: 29/3/2021

Conflicto de interés: los autores declaran que no existe conflicto de interés.

\section{CASO CLÍNICO}

Una paciente de 30 años, sin antecedentes patológicos, consultó por la presencia de múltiples máculas hipopigmentadas, redondeadas y asintomáticas, de 10 años de evolución. Estas, de contornos difusos y sensibilidad conservada, se localizaban en la zona proximal de ambos miembros inferiores y el tronco, donde confluían hacia la línea media (Foto 1). Antes, en 2015, había realizado tratamiento para la pitiriasis versicolor con itraconazol y, dos años después, con fluconazol, sin respuesta favorable. Se realizaron estudios de laboratorio, que incluyeron hemograma, función renal, hepatograma y perfil lipídico y tiroideo, los cuales no arrojaron hallazgos patológicos. El examen directo con luz de Wood fue negativo para $M$. furfur y $C$. acnes. El 
estudio de la biopsia de piel realizada en sacabocados (punch) informó: ausencia de elementos micóticos en el estrato córneo y disminución leve del número de melanocitos y de pigmento melánico en la capa basal epidérmica, en contraste con la piel normal adyacente (Foto 2). En la dermis, se observó un infiltrado inflamatorio mononuclear perivascular superficial leve y vasodilatación (Foto 3).

Con diagnóstico de HMP, se inició tratamiento con peróxido de benzoílo y clindamicina tópicos, pero debió suspenderse por la aparición de una dermatitis irritativa de contacto. Se decidió iniciar tratamiento con minociclina $100 \mathrm{mg} /$ día por vía oral durante 8 meses y se observó la disminución progresiva de las lesiones hasta su resolución total y sin recidiva desde hace 2 años (Foto 4).
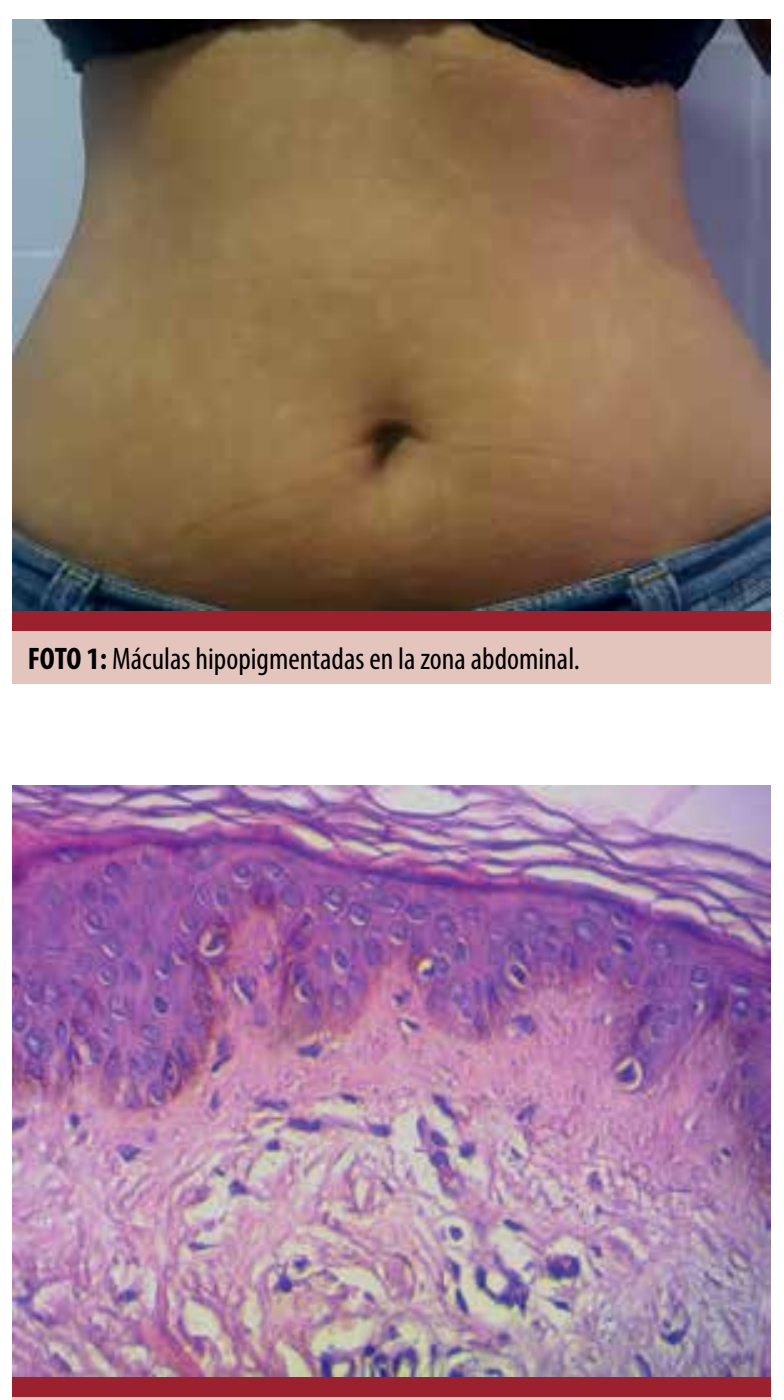

F0T0 2: Leve hipopigmentación melánica basal en la epidermis hacia la derecha, en comparación con una pigmentación melánica normal en la mitad izquierda de la fotografía (HyE, 400X).

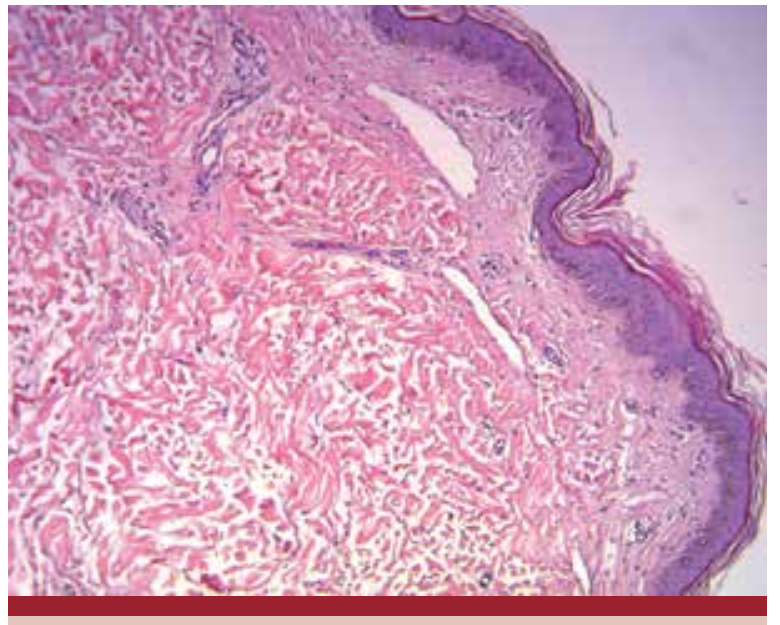

FOTO 3: Dermis con algunas telangiectasias y leve infiltrado inflamatorio mononuclear perivascular (HyE, 50X).

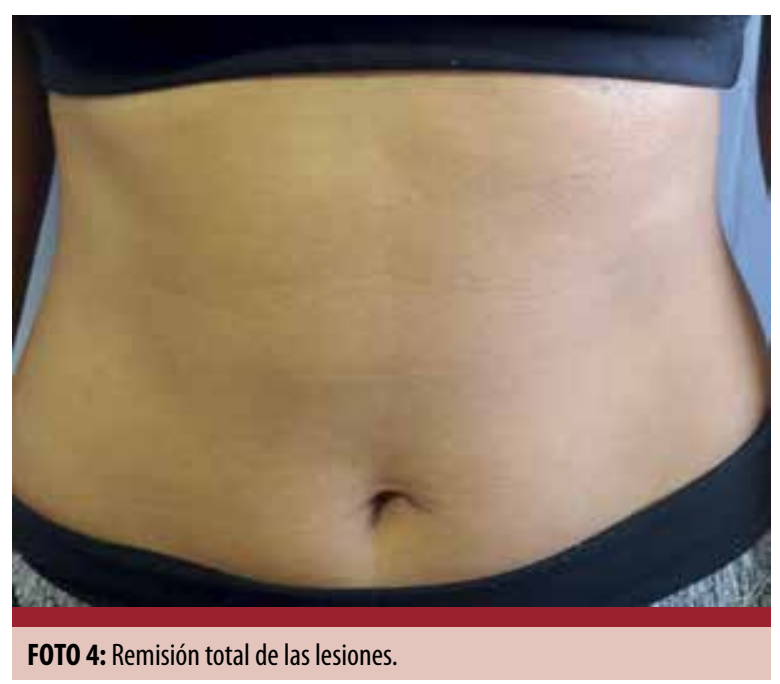

\section{COMENTARIOS}

La HMP es una dermatosis caracterizada por máculas hipopigmentadas redondeadas, de contorno difuso, asintomáticas y simétricas, con tendencia a confluir en la línea media, localizadas con mayor frecuencia en el tronco. No hay antecedentes de una dermatosis. Ocurre en todas las razas y tiene una distribución mundial, pero se identifica con mayor frecuencia en los adultos jóvenes, en el sexo femenino y en las personas con fototipos III y IV ${ }^{1,2}$.

Se cree que la microbiota de la piel, en particular la bacteria $C$. acnes, desempeña algún papel. Este comensal habita en las glándulas sebáceas y en los folículos pilosos de la piel y se ha asociado a ciertas dermatosis inflamatorias, incluida la $\mathrm{HMP}^{3}$. Mediante el análisis genómico de la bacteria, se identificaron seis grupos filogenéticos: IA1, IA2, IB, IC, II y III. Se ha demostrado un pre- 
dominio claro del filotipo III en el cultivo de la piel lesionada de los pacientes que presentan esta patología y su desarrollo estaría relacionado con el desequilibrio de la flora bacteriana dentro de los mismos subgrupos de $C$. acnes, con pérdida de la diversidad de estos y sobrerrepresentación de un solo filotipo. Esto llevaría a la producción de un factor despigmentante o un agente que interfiere en la melanogénesis y que ocasionaría la aparición de las máculas hipopigmentadas ${ }^{3-7}$.

El examen directo con luz de Wood puede mostrar, en ciertos pacientes, una fluorescencia folicular puntiforme, de color rojo anaranjado, causada por especies de $C$. acnes productoras de porfirinas cuando hay una densidad mínima de 1.000 microorganismos por centímetro cuadrado y cuando las lesiones están colonizadas por filotipos mixtos, que incluyen el subtipo I, el cual origina la fluorescencia, a diferencia de los subtipos II y III7-9. En el caso de la paciente, el resultado fue negativo, lo que podría indicar una colonización dominante o exclusiva por cepas de tipo III.

El tratamiento con tetraciclinas por vía oral o tópica se asocia a una reducción considerable en la proporción de $C$. acnes de tipo III, restaura el equilibrio en la distribución de filotipos y da como resultado una mejoría clínica ${ }^{1,2,7}$. Otros esquemas terapéuticos exitosos incluyen el uso de fototerapia UVA o UVB de

\section{BIBLIOGRAFÍA}

1. Santos JB, Almeida OL, Da Silva ML, Barreto ER. Efficacy of topical combination of benzoyl peroxide $5 \%$ and clindamycin $1 \%$ for the treatment of progressive macular hypomelanosis: a randomized, double-blind, placebo-controlled trial. An Bras Dermatol 2011;86:0-54.

2. Saleem MD, Oussedik E, Picardo M, Schoch JJ. Acquired disorders with hypopigmentation: A clinical approach to diagnosis and treatment. J Am Acad Dermatol 2019;80:12331250.

3. Petersen RLW, Scholz CFP, Jensen A, Brüggemann H, et ál. Propionibacterium Acnes phylogenetic type III is associated with progressive macular hypomelanosis. Eur J Microbiol Immunol 2017;7:37-45.

4. Westerhof W, Relyveld GN, Kingswijk M, Man P, et ál. Propionibacterium acnes and the pathogenesis of progressive macular hypomelanosis. Arch Dermatol 2004;140:210-214.

5. Corvec S, Dagnelie MA, Khammari A, Dréno B. Taxonomy and phylogeny of Cutibacterium (formerly Propionibacterium) acnes in inflammatory skin diseases. Ann Dermatol Venereol 2019;146:26-30. banda estrecha, como monoterapia o en combinación con antimicrobianos tópicos u orales ${ }^{2,7,10}$.

La HMP es una patología que suele subdiagnosticarse porque se la considera una pitiriasis versicolor, como sucedió en este caso clínico. En la variante hipopigmentada de esta última, las máculas son irregulares, asimétricas, bien definidas, con descamación al raspado (signo de la uñada positivo) y suelen ubicarse en el cuello y la parte superior del tronco. Otras enfermedades que deben tenerse en cuenta son la pitiriasis alba, con una frecuente afectación del rostro en los pacientes con dermatitis atópica; la discromía posinflamatoria, que puede producir un cuadro similar -aunque siempre existirá el antecedente de una dermatosis- $y$, por último, la variante hipopigmentada de la micosis fungoide, un linfoma cutáneo de células $\mathrm{T}$ con predominio en la infancia y la adolescencia, también común en los pacientes con fototipos altos.

Consideramos pertinente incluir la HMP en el diagnóstico diferencial de las lesiones hipopigmentadas en personas jóvenes, principalmente de sexo femenino, a fin de evitar procedimientos y tratamientos innecesarios. Destacamos la excelente respuesta clínica a la minociclina, avalada por la más reciente hipótesis del papel de C. acnes de tipo III en la patogenia de esta afección.

6. Barnard E, Liu J, Yankova E, Cavalcanti SM, et ál. Strains of the Propionibacterium acnes type III lineage are associated with the skin condition progressive macular hypomelanosis. Sci Rep 2016;6:1-9.

7. McDowell A, McLaughlin J, Layton AM. Is Cutibacterium (previously Propionibacterium) acnes a potential pathogenic factor in the aetiology of the skin disease progressive macular hypomelanosis? J Eur Acad Dermatol Venereol 2021;35:338-344.

8. Pflederer RT, Wuennenberg JP, Foote C, Aires D. Use of Wood's lamp to diagnose progressive macular hypomelanosis. J Am Acad Dermatol 2017;77:e99-e100.

9. Barnard E, Johson T, Ngo T, Arora U, et ál. Porphyrin production and regulation in cutaneous Propionibacteria. mSphere 2020;5:e00793-19.

10. Kim MB, Kim GW, Cho HH, Park HJ, et ál. Narrowband UVB treatment of progressive macular hypomelanosis. J Am Acad Dermatol 2012;66:598-605. 